\title{
Quantum integral inequalities on finite intervals
}

\author{
Jessada Tariboon ${ }^{1 *}$ and Sotiris K Ntouyas ${ }^{2}$
}

${ }^{*}$ Correspondence:
jessadat@kmutnb.ac.th
${ }^{1}$ Department of Mathematics,
Faculty of Applied Science, King
Mongkut's University of Technology
North Bangkok, Bangkok, Thailand
Full list of author information is
available at the end of the article

available at the end of the article

\begin{abstract}
In this paper, some of the most important integral inequalities of analysis are extended to quantum calculus. These include the Hölder, Hermite-Hadamard, trapezoid, Ostrowski, Cauchy-Bunyakovsky-Schwarz, Grüss, and Grüss-Čebyšev integral inequalities. The analysis relies on the notions of $q$-derivative and $q$-integral on finite intervals introduced by the authors in (Tariboon and Ntouyas in Adv. Differ. Equ. 2013:282, 2013).

MSC: 34A08; 26D10;26D15
\end{abstract}

Keywords: $q$-integral inequalities; Hölder's inequality; Hermite-Hadamard's inequality; Ostrowski's inequality; Grüss-Čebyšev integral inequality

\section{Introduction}

The integral inequalities play a fundamental role in the theory of differential equations. The study of the fractional $q$-integral inequalities is also of great importance. Integral inequalities have been studied extensively by several researchers either in classical analysis or in the quantum one; see [1-6] and references cited therein.

The purpose of this paper is to find $q$-calculus analogs of some classical integral inequalities. In particular, we will find $q$-generalizations of the Hölder, Hermite-Hadamard, trapezoid, Ostrowski, Cauchy-Bunyakovsky-Schwarz, Grüss, and Grüss-Čebyšev integral inequalities.

The paper is organized as follows: In Section 2, we shall introduce some definitions and auxiliary results which will help us to prove our main results. In Section 3, we establish our main results.

To the best of our knowledge, this paper is the first one that focuses on quantum integral inequalities on finite intervals.

\section{Preliminaries and auxiliary results}

Let $J:=[a, b] \subset \mathbb{R}$ be an interval and $0<q<1$ be a constant. We define $q$-derivative of a function $f: J \rightarrow \mathbb{R}$ at a point $x \in J$ on $[a, b]$ as follows.

Definition 2.1 Assume $f: J \rightarrow \mathbb{R}$ is a continuous function and let $x \in J$. Then the expression

$$
{ }_{a} D_{q} f(x)=\frac{f(x)-f(q x+(1-q) a)}{(1-q)(x-a)}, \quad x \neq a, \quad{ }_{a} D_{q} f(a)=\lim _{x \rightarrow a}{ }_{a} D_{q} f(x),
$$

is called the $q$-derivative on $J$ of function $f$ at $x$.

\section{Springer}

(02014 Tariboon and Ntouyas; licensee Springer. This is an Open Access article distributed under the terms of the Creative Commons Attribution License (http://creativecommons.org/licenses/by/2.0), which permits unrestricted use, distribution, and reproduction in any medium, provided the original work is properly cited. 
We say that $f$ is $q$-differentiable on $J$ provided ${ }_{a} D_{q} f(x)$ exists for all $x \in J$. Note that if $a=0$ in (2.1), then ${ }_{0} D_{q} f=\mathcal{D}_{q} f$, where $\mathcal{D}_{q}$ is the well-known $q$-derivative of the function $f(x)$ defined by

$$
\mathcal{D}_{q} f(x)=\frac{f(x)-f(q x)}{(1-q) x}
$$

For more details, see [7].

In addition, we should define the higher $q$-derivative of functions on $J$.

Definition 2.2 Let $f: J \rightarrow \mathbb{R}$ is a continuous function. We define the second-order $q$-derivative on interval $J$, which denoted as ${ }_{a} D_{q}^{2} f$, provided ${ }_{a} D_{q} f$ is $q$-differentiable on $J$ with ${ }_{a} D_{q}^{2} f={ }_{a} D_{q}\left({ }_{a} D_{q} f\right): J \rightarrow \mathbb{R}$. Similarly, we define higher order $q$-derivative on $J$, ${ }_{a} D_{q}^{n}: J_{k} \rightarrow \mathbb{R}$.

Example 2.1 Let $x \in[a, b]$ and $0<q<1$. Then, for $x \neq a$, we have

$$
\begin{aligned}
{ }_{a} D_{q} x^{2} & =\frac{x^{2}-(q x+(1-q) a)^{2}}{(1-q)(x-a)} \\
& =\frac{(1+q) x^{2}-2 q a x-(1-q) a^{2}}{x-a} \\
& =(1+q) x+(1-q) a .
\end{aligned}
$$

For $x=a$, we have $\lim _{x \rightarrow a}\left({ }_{a} D_{q} x^{2}\right)=2 a$.

Lemma 2.1 [8] Let $\alpha \in \mathbb{R}$, then we have

$$
{ }_{a} D_{q}(x-a)^{\alpha}=\left(\frac{1-q^{\alpha}}{1-q}\right)(x-a)^{\alpha-1} \text {. }
$$

The $q$-integral on interval $J$ is defined as follows.

Definition 2.3 Assume $f: J \rightarrow \mathbb{R}$ is a continuous function. Then the $q$-integral on $J$ is defined by

$$
\int_{a}^{x} f(t)_{a} d_{q} t=(1-q)(x-a) \sum_{n=0}^{\infty} q^{n} f\left(q^{n} x+\left(1-q^{n}\right) a\right)
$$

for $x \in J$. Moreover, if $c \in(a, x)$ then the definite $q$-integral on $J$ is defined by

$$
\begin{aligned}
\int_{c}^{x} f(t){ }_{a} d_{q} t= & \int_{a}^{x} f(t){ }_{a} d_{q} t-\int_{a}^{c} f(t){ }_{a} d_{q} t \\
= & (1-q)(x-a) \sum_{n=0}^{\infty} q^{n} f\left(q^{n} x+\left(1-q^{n}\right) a\right) \\
& -(1-q)(c-a) \sum_{n=0}^{\infty} q^{n} f\left(q^{n} c+\left(1-q^{n}\right) a\right) .
\end{aligned}
$$

Note that if $a=0$, then (2.4) reduces to the classical $q$-integral of a function $f(x)$, defined by $\int_{0}^{x} f(t)_{0} d_{q} t=(1-q) x \sum_{n=0}^{\infty} q^{n} f\left(q^{n} x\right)$ for $x \in[0, \infty)$. For more details, see [7]. 
Example 2.2 Let $f(x)=x$ for $x \in J$, then we have

$$
\begin{aligned}
\int_{a}^{x} f(t){ }_{a} d_{q} t & =\int_{a}^{x} t_{a} d_{q} t \\
& =(1-q)(x-a) \sum_{n=0}^{\infty} q^{n}\left(q^{n} x+\left(1-q^{n}\right) a\right) \\
& =\frac{(x-a)(x+q a)}{1+q} .
\end{aligned}
$$

Example 2.3 Let a constant $c \in J$, then we have

$$
\begin{aligned}
\int_{c}^{b}(t-c)_{a} d_{q} t & =\int_{a}^{b}(t-c)_{a} d_{q} t-\int_{a}^{c}(t-c)_{a} d_{q} t \\
& =\left[\frac{(t-a)(t+q a)}{1+q}-c t\right]_{a}^{b}-\left[\frac{(t-a)(t+q a)}{1+q}-c t\right]_{a}^{c} \\
& =\frac{b^{2}-(1+q) b c+q c^{2}}{1+q}-\frac{a(1-q)(b-c)}{1+q} .
\end{aligned}
$$

Note that if $q \rightarrow 1$, then (2.5) reduces to the classical integration

$$
\int_{c}^{b}(t-c) d t=\frac{(b-c)^{2}}{2}
$$

Theorem 2.1 [8] Let $f: J \rightarrow \mathbb{R}$ be a continuous function. Then we have:

(i) ${ }_{a} D_{q} \int_{a}^{x} f(t){ }_{a} d_{q} t=f(x)$;

(ii) $\int_{c}^{x}{ }_{a} D_{q} f(t){ }_{a} d_{q} t=f(x)-f(c)$ for $c \in(a, x)$.

Theorem 2.2 [8] Assume $f, g: J \rightarrow \mathbb{R}$ are continuous functions, $\alpha \in \mathbb{R}$. Then, for $x \in J$,

(i) $\int_{a}^{x}[f(t)+g(t)]_{a} d_{q} t=\int_{a}^{x} f(t)_{a} d_{q} t+\int_{a}^{x} g(t)_{a} d_{q} t$;

(ii) $\int_{a}^{x}(\alpha f)(t)_{a} d_{q} t=\alpha \int_{a}^{x} f(t)_{a} d_{q} t$;

(iii) $\int_{c}^{x} f(t)_{a} D_{q} g(t)_{a} d_{q} t=\left.(f g)\right|_{c} ^{x}-\int_{c}^{x} g(q t+(1-q) a)_{a} D_{q} f(t)_{a} d_{q} t$ for $c \in(a, x)$.

For the basic properties of $q$-derivative and $q$-integral on finite intervals, we refer to [8].

Lemma 2.2 For $\alpha \in \mathbb{R} \backslash\{-1\}$, the following formula holds:

$$
\int_{a}^{x}(t-a)^{\alpha}{ }_{a} d_{q} t=\left(\frac{1-q}{1-q^{\alpha+1}}\right)(x-a)^{\alpha+1} .
$$

Proof Let $f(x)=(x-a)^{\alpha+1}, x \in J$ and $\alpha \in \mathbb{R} \backslash\{-1\}$, then, by Definition 2.1, we have

$$
\begin{aligned}
{ }_{a} D_{q} f(x) & =\frac{(x-a)^{\alpha+1}-(q x+(1-q) a-a)^{\alpha+1}}{(1-q)(x-a)} \\
& =\frac{(x-a)^{\alpha+1}-q^{\alpha+1}(x-a)^{\alpha+1}}{(1-q)(x-a)} \\
& =\left(\frac{1-q^{\alpha+1}}{1-q}\right)(x-a)^{\alpha} .
\end{aligned}
$$

Applying $q$-integral on $J$ for (2.7), we obtain (2.6) as required. 
Example 2.4 Let $x \in[a, b]$ and $0<q<1$. Then, from $q$-integrating by parts and Lemmas 2.1 and 2.2, we have

$$
\begin{aligned}
\int_{a}^{x} t(t-a)_{a} d_{q} t & =\frac{1}{1+q} \int_{a}^{x} t_{a} D_{q}(t-a)^{2}{ }_{a} d_{q} t \\
& =\frac{1}{1+q}\left[\left.t(t-a)^{2}\right|_{a} ^{x}-\int_{a}^{x}(q t+(1-q) a-a)^{2}{ }_{a} d_{q} t\right] \\
& =\frac{1}{1+q}\left[x(x-a)^{2}-q^{2} \int_{a}^{x}(t-a)^{2}{ }_{a} d_{q} t\right] \\
& =\frac{1}{1+q}\left[x(x-a)^{2}-\frac{q^{2}(x-a)^{3}}{1+q+q^{2}}\right] \\
& =\frac{(x-a)^{2}}{1+q}\left[\frac{x(1+q)+q^{2} a}{1+q+q^{2}}\right] .
\end{aligned}
$$

\section{Quantum integral inequalities on finite intervals}

In this section, some of the most important integral inequalities of analysis are extended to quantum calculus. We start with the $q$-Hölder inequality on the interval $J=[a, b]$.

Theorem 3.1 Let $x \in J, 0<q<1, p_{1}, p_{2}>1$ such that $\frac{1}{p_{1}}+\frac{1}{p_{2}}=1$. Then we have

$$
\int_{a}^{x}|f(t)||g(t)|{ }_{a} d_{q} t \leq\left(\int_{a}^{x}|f(t)|^{p_{1}}{ }_{a} d_{q} t\right)^{\frac{1}{p_{1}}}\left(\int_{a}^{x}|g(t)|^{p_{2}}{ }_{a} d_{q} t\right)^{\frac{1}{p_{2}}} .
$$

Proof From Definition 2.3 and the discrete Hölder inequality, we have

$$
\begin{aligned}
& \int_{a}^{x}|f(t)||g(t)|{ }_{a} d_{q} t \\
& =(1-q)(x-a) \sum_{n=0}^{\infty} q^{n}\left|\left(q^{n} x+\left(1-q^{n}\right) a\right)\right|\left|g\left(q^{n} x+\left(1-q^{n}\right) a\right)\right| \\
& =(1-q)(x-a) \sum_{n=0}^{\infty}\left(\left|f\left(q^{n} x+\left(1-q^{n}\right) a\right)\right|\left(q^{n}\right)^{\frac{1}{p_{1}}}\right) \\
& \quad \times\left(\left|g\left(q^{n} x+\left(1-q^{n}\right) a\right)\right|\left(q^{n}\right)^{\frac{1}{p_{2}}}\right) \\
& \leq \quad\left((1-q)(x-a) \sum_{n=0}^{\infty}\left|f\left(q^{n} x+\left(1-q^{n}\right) a\right)\right|^{p_{1}} q^{n}\right)^{\frac{1}{p_{1}}} \\
& \quad \times\left((1-q)(x-a) \sum_{n=0}^{\infty}\left|g\left(q^{n} x+\left(1-q^{n}\right) a\right)\right|^{p_{2}} q^{n}\right)^{\frac{1}{p_{2}}} \\
& =\quad\left(\int_{a}^{x}|f(t)|^{p_{1}}{ }_{a} d_{q} t\right)^{\frac{1}{p_{1}}}\left(\int_{a}^{x}|g(t)|^{p_{2}}{ }_{a} d_{q} t\right)^{\frac{1}{p_{2}}} \cdot
\end{aligned}
$$

Therefore, inequality (3.1) is valid.

Remark 3.1 If $a=0$, then inequality (3.1) reduces to the classical $q$-Hölder inequality in [1, p.604]. 
Next, we present the $q$-Hermite-Hadamard integral inequality on $[a, b]$.

Theorem 3.2 Let $f: J \rightarrow \mathbb{R}$ be a convex continuous function on $J$ and $0<q<1$. Then we have

$$
f\left(\frac{a+b}{2}\right) \leq \frac{1}{b-a} \int_{a}^{b} f(t)_{a} d_{q} t \leq \frac{q f(a)+f(b)}{1+q} .
$$

Proof The convexity of $f$ on $[a, b]$ means that

$$
f((1-t) a+t b) \leq(1-t) f(a)+t f(b)
$$

for all $t \in[0,1]$.

Taking $q$-integration for (3.3) over $t$ on $[0,1]$, we have

$$
\int_{0}^{1} f((1-t) a+t b){ }_{0} d_{q} t \leq f(a) \int_{0}^{1}(1-t)_{0} d_{q} t+f(b) \int_{0}^{1} t_{0} d_{q} t .
$$

From Example 2.2, we have

$$
\int_{0}^{1} t_{0} d_{q} t=\frac{1}{1+q} \text { and } \int_{0}^{1}(1-t)_{0} d_{q} t=\frac{q}{1+q} .
$$

Definition of $q$-integration on $J$ leads to

$$
\begin{aligned}
\int_{0}^{1} f((1-t) a+t b){ }_{0} d_{q} t & =(1-q) \sum_{n=0}^{\infty} q^{n} f\left(\left(1-q^{n}\right) a+q^{n} b\right) \\
& =\frac{(1-q)(b-a)}{(b-a)} \sum_{n=0}^{\infty} q^{n} f\left(\left(1-q^{n}\right) a+q^{n} b\right) \\
& =\frac{1}{b-a} \int_{a}^{b} f(t)_{a} d_{q} t,
\end{aligned}
$$

which gives the second part of (3.2) by using (3.4).

To prove the first part of (3.2), we use the convex property of $f$ as follows:

$$
\begin{aligned}
\frac{1}{2}[f((1-t) a+t b)+f(t a+(1-t) b)] & \geq f\left(\frac{(1-t) a+t b+t a+(1-t) b}{2}\right) \\
& =f\left(\frac{a+b}{2}\right) .
\end{aligned}
$$

Again $q$-integrating to the above inequality over $t$ on $[0,1]$ and changing variables, we get

$$
\begin{aligned}
f\left(\frac{a+b}{2}\right) & \leq \frac{1}{2}\left[\int_{0}^{1} f((1-t) a+t b)_{0} d_{q} t+\int_{0}^{1} f(t a+(1-t) b)_{0} d_{q} t\right] \\
& =\frac{1}{b-a} \int_{a}^{b} f(t)_{a} d_{q} t .
\end{aligned}
$$

The proof is completed. 
Remark 3.2 If $q \rightarrow 1$, then inequality (3.2) reduces to the Hermite-Hadamard integral inequality

$$
f\left(\frac{a+b}{2}\right) \leq \frac{1}{b-a} \int_{a}^{b} f(t) d t \leq \frac{f(a)+f(b)}{2} .
$$

See also $[3,9]$.

Next comes the $q$-trapezoid inequality on the interval $J=[a, b]$. We use the notation $\|\cdot\|$ for the usual supremum norm on $[a, b]$.

Theorem 3.3 Let $f: J \rightarrow \mathbb{R}$ be a q-differentiable function with ${ }_{a} D_{q} f$ continuous on $[a, b]$ and $0<q<1$. Then we have

$$
\left|\int_{a}^{b} f(q t+(1-q) a){ }_{a} d_{q} t-(b-a)\left(\frac{f(b)+f(a)}{2}\right)\right| \leq \frac{(b-a)^{2}}{2(1+q)}\left\|_{a} D_{q} f\right\| .
$$

Proof The $q$-integration by parts on interval $J$ gives

$$
\begin{aligned}
\int_{a}^{b}\left(t-\frac{a+b}{2}\right){ }_{a} D_{q} f(t){ }_{a} d_{q} t= & (b-a)\left(\frac{f(b)+f(a)}{2}\right) \\
& -\int_{a}^{b} f(q t+(1-q) a)_{a} d_{q} t .
\end{aligned}
$$

Using the properties of modulus for (3.6), we obtain

$$
\begin{aligned}
& \left|\int_{a}^{b} f(q t+(1-q) a){ }_{a} d_{q} t-(b-a)\left(\frac{f(b)+f(a)}{2}\right)\right| \\
& \quad \leq\left.\int_{a}^{b}\left|t-\frac{a+b}{2}\right|{ }_{a} D_{q} f(t)\right|_{a} d_{q} t \\
& \quad \leq\left\|_{a} D_{q} f\right\| \int_{a}^{b}\left|t-\frac{a+b}{2}\right|{ }_{a} d_{q} t .
\end{aligned}
$$

Applying Examples 2.2 and 2.3, we have

$$
\begin{aligned}
\int_{a}^{b}\left|t-\frac{a+b}{2}\right|{ }_{a} d_{q} t= & \int_{a}^{\frac{a+b}{2}}\left(\frac{a+b}{2}-t\right){ }_{a} d_{q} t+\int_{\frac{a+b}{2}}^{b}\left(t-\frac{a+b}{2}\right){ }_{a} d_{q} t \\
= & \left(\frac{a+b}{2}\right)\left(\frac{b-a}{2}\right)-\left(\frac{b-a}{4}\right)\left(\frac{(1+2 q) a+b}{1+q}\right) \\
& +\frac{b^{2}-b(1+q)((a+b) / 2)+q((a+b) / 2)^{2}}{1+q} \\
& -a\left(\frac{1-q}{2}\right)\left(\frac{b-a}{1+q}\right) \\
= & \frac{(b-a)^{2}}{2(1+q)} .
\end{aligned}
$$

Combining (3.7) and (3.8), we obtain inequality (3.5) as required. 
Remark 3.3 If $q \rightarrow 1$, then inequality (3.5) reduces to the well-known trapezoid inequality as

$$
\left|\int_{a}^{b} f(t) d t-(b-a)\left(\frac{f(b)+f(a)}{2}\right)\right| \leq \frac{(b-a)^{2}}{4}\left\|f^{\prime}\right\| .
$$

See also $[3,9]$.

The next theorem deals with the $q$-trapezoid inequality with second-order $q$-derivative on $[a, b]$.

Theorem 3.4 Let $f: J \rightarrow \mathbb{R}$ be a twice q-differentiable function with ${ }_{a} D_{q}^{2} f$ continuous on $[a, b]$ and $0<q<1$. Then we have

$$
\begin{aligned}
& \left|\int_{a}^{b} f\left(q^{2} t+\left(1-q^{2}\right) a\right){ }_{a} d_{q} t-\frac{(b-a)}{1+q}(q f(q b+(1-q) a)+f(a))\right| \\
& \quad \leq \frac{q^{2}(b-a)^{3}}{(1+q)^{2}\left(1+q+q^{2}\right)}\left\|_{a} D_{q}^{2} f\right\| .
\end{aligned}
$$

Proof The $q$-integration by parts on interval $J$ two-times and taking into account Example 2.1, we have

$$
\begin{aligned}
\int_{a}^{b}(t-a)(b-t)_{a} D_{q}^{2} f(t)_{a} d_{q} t \\
=-\int_{a}^{b}(q a+b-(1+q) t)_{a} D_{q} f(q t+(1-q) a)_{a} d_{q} t \\
=[-(q a+b-(1+q) t) f(q t+(1-q) a)]_{a}^{b} \\
\quad+\int_{a}^{b} f\left(q^{2} t+\left(1-q^{2}\right) a\right)_{a} D_{q}(q a+b-(1+q) t)_{a} d_{q} t \\
=q(b-a) f(q b+(1-q) a)+(b-a) f(a) \\
\quad-(1+q) \int_{a}^{b} f\left(q^{2} t+\left(1-q^{2}\right) a\right){ }_{a} d_{q} t .
\end{aligned}
$$

Therefore,

$$
\begin{aligned}
& \left|\int_{a}^{b} f\left(q^{2} t+\left(1-q^{2}\right) a\right)_{a} d_{q} t-\frac{(b-a)}{1+q}(q f(q b+(1-q) a)+f(a))\right| \\
& \quad \leq\left.\left.\frac{1}{1+q} \int_{a}^{b}(t-a)(b-t)\right|_{a} D_{q}^{2} f(t)\right|_{a} d_{q} t \\
& \quad \leq \frac{\left\|_{a} D_{q}^{2} f\right\|}{1+q} \int_{a}^{b}(t-a)(b-t)_{a} d_{q} t .
\end{aligned}
$$

Since

$$
\int_{a}^{b}(t-a)(b-t){ }_{a} d_{q} t=b \int_{a}^{b}(t-a)_{a} d_{q} t-\int_{a}^{b} t(t-a)_{a} d_{q} t
$$


from Lemma 2.2 and Example 2.4, we have

$$
\begin{aligned}
\int_{a}^{b}(t-a)(b-t)_{a} d_{q} t & =\frac{b}{1+q}(b-a)^{2}-\frac{(b-a)^{2}}{1+q}\left[\frac{b(1+q)+q^{2} a}{1+q+q^{2}}\right] \\
& =\frac{q^{2}(b-a)^{3}}{(1+q)\left(1+q+q^{2}\right)} .
\end{aligned}
$$

Combining (3.10) and (3.11), we deduce that inequality (3.9) is valid.

Remark 3.4 If $q \rightarrow 1$, then inequality (3.9) reduces to the trapezoid inequality in terms of the second derivative as

$$
\left|\int_{a}^{b} f(t) d t-\frac{(b-a)}{2}(f(b)+f(a))\right| \leq \frac{(b-a)^{3}}{12}\left\|f^{\prime \prime}\right\| .
$$

See also $[3,9]$.

In the following theorem we establish the $q$-Ostrowski integral inequality on interval $J$.

Theorem 3.5 Let $f: J \rightarrow \mathbb{R}$ be a q-differentiable function with ${ }_{a} D_{q} f$ continuous on $[a, b]$ and $0<q<1$. Then we have

$$
\begin{aligned}
& \left|f(x)-\frac{1}{b-a} \int_{a}^{b} f(t){ }_{a} d_{q} t\right| \\
& \quad \leq\left\|_{a} D_{q} f\right\|(b-a)\left[\frac{2 q}{1+q}\left(\frac{x-\frac{(3 q-1) a+(1+q) b}{4 q}}{b-a}\right)^{2}+\frac{\left(-q^{2}+6 q-1\right)}{8 q(1+q)}\right] .
\end{aligned}
$$

Proof Applying the Lagrangian mean value theorem [10], for $x, t \in J$, it follows that

$$
\begin{aligned}
\left|f(x)-\frac{1}{b-a} \int_{a}^{b} f(t)_{a} d_{q} t\right| & =\left|\frac{1}{b-a} \int_{a}^{b}(f(x)-f(t))_{a} d_{q} t\right| \\
& \leq \frac{1}{b-a} \int_{a}^{b}|f(x)-f(t)|_{a} d_{q} t \\
& \leq \frac{\left\|_{a} D_{q} f\right\|}{b-a} \int_{a}^{b}|x-t|_{a} d_{q} t \\
& =\frac{\left\|_{a} D_{q} f\right\|}{b-a}\left[\int_{a}^{x}(x-t)_{a} d_{q} t+\int_{x}^{b}(t-x)_{a} d_{q} t\right] .
\end{aligned}
$$

Taking into account Examples 2.2 and 2.3, for $x, t \in J$, we obtain

$$
\begin{aligned}
& \int_{a}^{x}(x-t)_{a} d_{q} t+\int_{x}^{b}(t-x){ }_{a} d_{q} t \\
& \quad=\left[\frac{q x^{2}-2 q a x+q a^{2}}{1+q}\right]+\left[\frac{b^{2}-(1+q) b x+q x^{2}}{1+q}-\frac{a(1-q)(b-x)}{1+q}\right] \\
& =\frac{2 q}{1+q}\left[x^{2}-\left(\frac{(3 q-1) a+(1+q) b}{2 q}\right) x\right]+\frac{q a^{2}+b^{2}-(1-q) a b}{1+q} \\
& =\frac{2 q}{1+q}\left(x-\frac{(3 q-1) a+(1+q) b}{4 q}\right)^{2}+\frac{\left(-q^{2}+6 q-1\right)}{(1+q) 8 q}(b-a)^{2} .
\end{aligned}
$$

The inequality (3.12) are obtained by combining (3.13) and (3.14). 
Remark 3.5 If $q \rightarrow 1$, then inequality (3.12) reduces to the classical Ostrowski integral inequality as

$$
\left|f(x)-\frac{1}{b-a} \int_{a}^{b} f(t) d t\right| \leq\left[\frac{1}{4}+\left(\frac{x-\frac{a+b}{2}}{b-a}\right)^{2}\right](b-a)\left\|f^{\prime}\right\| .
$$

See also $[3,9]$.

Let us prove the $q$-Korkine identity on interval $J$.

Lemma 3.1 Let $f, g: J \rightarrow \mathbb{R}$ be continuous functions on $J$ and $0<q<1$. Then we have

$$
\begin{aligned}
& \frac{1}{2} \int_{a}^{b} \int_{a}^{b}(f(x)-f(y))(g(x)-g(y))_{a} d_{q} x_{a} d_{q} y \\
& \quad=(b-a) \int_{a}^{b} f(x) g(x){ }_{a} d_{q} x-\left(\int_{a}^{b} f(x)_{a} d_{q} x\right)\left(\int_{a}^{b} g(x)_{a} d_{q} x\right) .
\end{aligned}
$$

Proof From Definition 2.3, we have

$$
\begin{aligned}
& \int_{a}^{b} \int_{a}^{b}(f(x)-f(y))(g(x)-g(y))_{a} d_{q} x_{a} d_{q} y \\
& =\int_{a}^{b} \int_{a}^{b}[f(x) g(x)-f(x) g(y)-f(y) g(x)+f(y) g(y)]_{a} d_{q} x_{a} d_{q} y \\
& =(1-q)(b-a) \sum_{n=0}^{\infty} q^{n} f\left(q^{n} b+\left(1-q^{n}\right) a\right) g\left(q^{n} b+\left(1-q^{n}\right) a\right)(b-a) \\
& \quad-(1-q)^{2}(b-a)^{2}\left(\sum_{n=0}^{\infty} q^{n} f\left(q^{n} b+\left(1-q^{n}\right) a\right)\right)\left(\sum_{n=0}^{\infty} q^{n} g\left(q^{n} b+\left(1-q^{n}\right) a\right)\right) \\
& \quad-(1-q)^{2}(b-a)^{2}\left(\sum_{n=0}^{\infty} q^{n} g\left(q^{n} b+\left(1-q^{n}\right) a\right)\right)\left(\sum_{n=0}^{\infty} q^{n} f\left(q^{n} b+\left(1-q^{n}\right) a\right)\right) \\
& \quad+(1-q)(b-a) \sum_{n=0}^{\infty} q^{n} f\left(q^{n} b+\left(1-q^{n}\right) a\right) g\left(q^{n} b+\left(1-q^{n}\right) a\right)(b-a) \\
& =2(b-a) \int_{a}^{b} f(x) g(x)_{a} d_{q x} x-2\left(\int_{a}^{b} f(x)_{a} d_{q} x\right)\left(\int_{a}^{b} g(x)_{a} d_{q} x\right),
\end{aligned}
$$

from which one deduces (3.15).

Now, we will prove the $q$-Cauchy-Bunyakovsky-Schwarz integral inequality for double integrals on $[a, b]$.

Lemma 3.2 Let $f, g: J \rightarrow \mathbb{R}$ be continuous functions on $J$ and $0<q<1$. Then we have

$$
\begin{aligned}
& \left|\int_{a}^{b} \int_{a}^{b} f(x, y) g(x, y)_{a} d_{q} x_{a} d_{q} y\right| \\
& \quad \leq\left[\int_{a}^{b} \int_{a}^{b} f^{2}(x, y)_{a} d_{q} x_{a} d_{q} y\right]^{\frac{1}{2}}\left[\int_{a}^{b} \int_{a}^{b} g^{2}(x, y)_{a} d_{q} x_{a} d_{q} y\right]^{\frac{1}{2}} .
\end{aligned}
$$


Proof According to Definition 2.3, we have the double $q$-integral on $J$ as

$$
\begin{aligned}
& \int_{a}^{b} \int_{a}^{b} f(x, y)_{a} d_{q} x_{a} d_{q} y \\
& \quad=\int_{a}^{b}\left((1-q)(b-a) \sum_{n=0}^{\infty} q^{n} f\left(q^{n} b+\left(1-q^{n}\right) a, y\right)\right){ }_{a} d_{q} y \\
& \quad=(1-q)^{2}(b-a)^{2} \sum_{n=0}^{\infty} \sum_{i=0}^{\infty} q^{n+i} f\left(q^{n} b+\left(1-q^{n}\right) a, q^{i} b+\left(1-q^{i}\right) a\right) .
\end{aligned}
$$

Applying the discrete Cauchy-Schwarz inequality, we have

$$
\begin{aligned}
\left(\int_{a}^{b} \int_{a}^{b} f(x, y) g(x, y)_{a} d_{q} x_{a} d_{q} y\right)^{2} \\
=\left((1-q)^{2}(b-a)^{2} \sum_{n=0}^{\infty} \sum_{i=0}^{\infty} q^{n+i} f\left(q^{n} b+\left(1-q^{n}\right) a, q^{i} b+\left(1-q^{i}\right) a\right)\right. \\
\left.\quad \times g\left(q^{n} b+\left(1-q^{n}\right) a, q^{i} b+\left(1-q^{i}\right) a\right)\right)^{2} \\
\left.\leq \quad(1-q)^{2}(b-a)^{2} \sum_{n=0}^{\infty} \sum_{i=0}^{\infty} q^{n+i} f^{2}\left(q^{n} b+\left(1-q^{n}\right) a, q^{i} b+\left(1-q^{i}\right) a\right)\right) \\
\quad \times\left((1-q)^{2}(b-a)^{2} \sum_{n=0}^{\infty} \sum_{i=0}^{\infty} q^{n+i} g^{2}\left(q^{n} b+\left(1-q^{n}\right) a, q^{i} b+\left(1-q^{i}\right) a\right)\right) \\
=\left(\int_{a}^{b} \int_{a}^{b} f^{2}(x, y)_{a} d_{q^{x}}{ }_{a} d_{q} y\right)\left(\int_{a}^{b} \int_{a}^{b} g^{2}(x, y)_{a} d_{q^{x}} x_{a} d_{q} y\right) .
\end{aligned}
$$

Therefore, inequality (3.16) is valid.

Remark 3.6 If $q \rightarrow 1$, then Lemmas 3.1 and 3.2 are reduced to the usual Korkine identity and Cauchy-Bunyakovsky-Schwarz integral inequality for double integrals, respectively. For more details, see [3] and [9].

We define the $q$-Čebyšev functional $T(f, g)$ on interval $J$ by

$$
\begin{aligned}
T(f, g)= & \frac{1}{b-a} \int_{a}^{b} f(x) g(x)_{a} d_{q} x \\
& -\left(\frac{1}{b-a} \int_{a}^{b} f(x)_{a} d_{q} x\right)\left(\frac{1}{b-a} \int_{a}^{b} g(x)_{a} d_{q} x\right) .
\end{aligned}
$$

By using Lemmas 3.1 and 3.2 coupled with (3.17), we obtain the $q$-Grüss integral inequality on interval $[a, b]$. The proof of the following theorem is similar to the classical Grüss integral inequality; see $[3,9]$. Therefore, we omit it.

Theorem 3.6 Let $f, g: J \rightarrow \mathbb{R}$ be continuous functions on $[a, b]$ and satisfy

$$
\phi \leq f(x) \leq \Phi, \quad \gamma \leq g(x) \leq \Gamma \quad \text { for all } x \in[a, b], \phi, \Phi, \gamma, \Gamma \in \mathbb{R}
$$


Then we have the inequality

$$
\begin{aligned}
& \left|\frac{1}{b-a} \int_{a}^{b} f(x) g(x){ }_{a} d_{q} x-\left(\frac{1}{b-a} \int_{a}^{b} f(x){ }_{a} d_{q} x\right)\left(\frac{1}{b-a} \int_{a}^{b} g(x){ }_{a} d_{q} x\right)\right| \\
& \quad \leq \frac{1}{4}(\Phi-\phi)(\Gamma-\gamma) .
\end{aligned}
$$

Remark 3.7 The inequality (3.19) is similar to $q$-Grüss integral inequality in [11]. However, the results from [11] obtained by using the restricted definite $q$-integral which is a finite sum as a special type of the definite $q$-integral.

Now, we are going to prove the $q$-Grüss-Čebyšev integral inequality on interval $[a, b]$.

Theorem 3.7 Let $f, g: J \rightarrow \mathbb{R}$ be $L_{1}, L_{2}$-Lipschitzian continuous functions on $[a, b]$, so that

$$
|f(x)-f(y)| \leq L_{1}|x-y|, \quad|g(x)-g(y)| \leq L_{2}|x-y|
$$

for all $x, y \in[a, b]$. Then we have the inequality

$$
\begin{aligned}
& \left|\frac{1}{b-a} \int_{a}^{b} f(x) g(x)_{a} d_{q} x-\left(\frac{1}{b-a} \int_{a}^{b} f(x){ }_{a} d_{q} x\right)\left(\frac{1}{b-a} \int_{a}^{b} g(x){ }_{a} d_{q} x\right)\right| \\
& \quad \leq \frac{q L_{1} L_{2}}{\left(1+q+q^{2}\right)(1+q)^{2}}(b-a)^{2} .
\end{aligned}
$$

Proof We recall the $q$-Korkine identity on interval $J$ as

$$
\begin{gathered}
(b-a) \int_{a}^{b} f(x) g(x){ }_{a} d_{q} x-\left(\int_{a}^{b} f(x)_{a} d_{q} x\right)\left(\int_{a}^{b} g(x)_{a} d_{q} x\right) \\
=\frac{1}{2} \int_{a}^{b} \int_{a}^{b}(f(x)-f(y))(g(x)-g(y))_{a} d_{q} x d_{a} d_{q} y .
\end{gathered}
$$

From (3.20), we get

$$
|(f(x)-f(y))(g(x)-g(y))| \leq L_{1} L_{2}(x-y)^{2}
$$

for all $x, y \in[a, b]$.

The double $q$-integration for (3.23) on $J \times J$ leads to

$$
\begin{aligned}
& \int_{a}^{b} \int_{a}^{b}|(f(x)-f(y))(g(x)-g(y))|{ }_{a} d_{q} x_{a} d_{q} y \\
& \quad \leq L_{1} L_{2} \int_{a}^{b} \int_{a}^{b}(x-y)^{2}{ }_{a} d_{q} x_{a} d_{q} y \\
& \quad=L_{1} L_{2} \int_{a}^{b} \int_{a}^{b}\left(x^{2}-2 x y+y^{2}\right)_{a} d_{q} x_{a} d_{q} y \\
& \quad=L_{1} L_{2}\left[2(b-a) \int_{a}^{b} x^{2}{ }_{a} d_{q} x-2\left(\int_{a}^{b} x_{a} d_{q} x\right)^{2}\right] .
\end{aligned}
$$


Indeed,

$$
\begin{aligned}
\int_{a}^{b} x^{2}{ }_{a} d_{q} x & =\int_{a}^{b}(x-a+a)^{2}{ }_{a} d_{q} x \\
& =\int_{a}^{b}(x-a)^{2}{ }_{a} d_{q} x+2 a \int_{a}^{b}(x-a)_{a} d_{q} x+a^{2} \int_{a}^{b}{ }_{a} d_{q} x \\
& =\frac{(b-a)^{3}}{1+q+q^{2}}+2 a \frac{(b-a)^{2}}{1+q}+a^{2}(b-a) \\
& =\frac{(b-a)\left((1+q) b^{2}+2 q^{2} a b+q\left(1+q^{2}\right) a^{2}\right)}{(1+q)\left(1+q+q^{2}\right)}
\end{aligned}
$$

Note that if $q \rightarrow 1$, then (3.25) reduces to the integral

$$
\int_{a}^{b} x^{2} d x=\frac{b^{3}-a^{3}}{3}
$$

By direct computation, we have

$$
(b-a) \int_{a}^{b} x^{2}{ }_{a} d_{q} x-\left(\int_{a}^{b} x_{a} d_{q} x\right)^{2}=\frac{q(b-a)^{4}}{\left(1+q+q^{2}\right)(1+q)^{2}} .
$$

Thus, from (3.24) and (3.26), we obtain

$$
\int_{a}^{b} \int_{a}^{b}|(f(x)-f(y))(g(x)-g(y))|{ }_{a} d_{q} x_{a} d_{q} y \leq \frac{2 q(b-a)^{4}}{\left(1+q+q^{2}\right)(1+q)^{2}} L_{1} L_{2} .
$$

Using (3.22), we obtain (3.21).

Remark 3.8 If $q \rightarrow 1$, then inequality (3.21) reduces to the classical Grüss-Čebyšev integral inequality as

$$
\begin{aligned}
& \left|\frac{1}{b-a} \int_{a}^{b} f(x) g(x) d x-\left(\frac{1}{b-a} \int_{a}^{b} f(x) d x\right)\left(\frac{1}{b-a} \int_{a}^{b} g(x) d x\right)\right| \\
& \quad \leq \frac{L_{1} L_{2}}{12}(b-a)^{2} .
\end{aligned}
$$

See also $[3,9]$.

\section{Competing interests}

The authors declare that they have no competing interests.

\section{Authors' contributions}

Both authors contributed equally in this article. They read and approved the final manuscript.

\section{Author details}

'Department of Mathematics, Faculty of Applied Science, King Mongkut's University of Technology North Bangkok, Bangkok, Thailand. ${ }^{2}$ Department of Mathematics, University of Ioannina, Ioannina, 451 10, Greece.

\section{Authors' information}

Sotiris K Ntouyas is a member of Nonlinear Analysis and Applied Mathematics (NAAM) - Research Group at King Abdulaziz University, Jeddah, Saudi Arabia. 


\section{Acknowledgements}

The research of J Tariboon is supported by King Mongkut's University of Technology North Bangkok, Thailand.

Received: 27 January 2014 Accepted: 14 March 2014 Published: 26 Mar 2014

\section{References}

1. Anastassiou, GA: Intelligent Mathematics: Computational Analysis. Springer, New York (2011)

2. Belarbi, S, Dahmani, Z: On some new fractional integral inequalities. JIPAM. J. Inequal. Pure Appl. Math. 10, Article ID 86 (2009)

3. Cerone, P, Dragomir, SS: Mathematical Inequalities. CRC Press, New York (2011)

4. Dahmani, Z: New inequalities in fractional integrals. Int. J. Nonlinear Sci. 9, 493-497 (2010)

5. Dragomir, SS: Some integral inequalities of Grüss type. Indian J. Pure Appl. Math. 31, 397-415 (2002)

6. Ogunmez, H, Ozkan, UM: Fractional quantum integral inequalities. J. Inequal. Appl. 2011, Article ID 787939 (2011)

7. Kac, V, Cheung, P: Quantum Calculus. Springer, New York (2002)

8. Tariboon, J, Ntouyas, SK: Quantum calculus on finite intervals and applications to impulsive difference equations. Adv. Differ. Equ. 2013, 282 (2013)

9. Pachpatte, BG: Analytic Inequalities. Atlantis Press, Paris (2012)

10. Florea, A, Niculescu, CP: A note on Ostrowski's inequality. J. Inequal. Appl. 2005(5), 459-468 (2005)

11. Gauchman, H: Integral inequalities in q-calculus. Comput. Math. Appl. 47, 281-300 (2004)

10.1186/1029-242X-2014-121

Cite this article as: Tariboon and Ntouyas: Quantum integral inequalities on finite intervals. Journal of Inequalities and Applications 2014, 2014:121

\section{Submit your manuscript to a SpringerOpen ${ }^{\ominus}$ journal and benefit from:}

- Convenient online submission

Rigorous peer review

- Immediate publication on acceptance

- Open access: articles freely available online

- High visibility within the field

- Retaining the copyright to your article 